\title{
Defibrillation via the Elimination of Spiral Turbulence in a Model for Ventricular Fibrillation
}

\author{
Sitabhra Sinha, ${ }^{1,2}$ Ashwin Pande, ${ }^{1}$ and Rahul Pandit ${ }^{1,2}$ \\ ${ }^{1}$ Centre for Condensed Matter Theory, Department of Physics, Indian Institute of Science, Bangalore 560 012, India \\ ${ }^{2}$ Condensed Matter Theory Unit, Jawaharlal Nehru Centre for Advanced Scientific Research, Bangalore 560 064, India
}

\begin{abstract}
Ventricular fibrillation, the major reason behind sudden cardiac death, is turbulent cardiac electrical activity in which rapid, irregular disturbances in the spatiotemporal electrical activation of the heart make it incapable of any concerted pumping action. Methods of controlling ventricular fibrillation include electrical defibrillation as well as injected medication. Electrical defibrillation, though widely used, involves subjecting the whole heart to massive, and often counterproductive, electrical shocks. We propose a defibrillation method that uses a very low-amplitude shock (of order $\mathrm{mV}$ ) applied for a brief duration (of order $100 \mathrm{~ms}$ ) and over a coarse mesh of lines on our model ventricle.
\end{abstract}

Ventricular fibrillation (VF), the leading cause of sudden cardiac death, is responsible for about one out of every six deaths in the U.S. [1,2]. In the absence of any attempt at defibrillation, VF leads to death in a few minutes. The importance of this problem can hardly be overemphasized, so it is not surprising that many studies of VF have been carried out in various mammalian hearts [1] and mathematical models [3]. Recent experiments [4,5] have shown that, though VF is turbulent cardiac activity, it is associated with the formation, and subsequent breakup, of electrophysiological structures that emit spiral waves; these are referred to as rotors or simply spirals. Such structures and their breakup have also been obtained in a set of partial differential equations of the Fitzhugh-Nagumo type; this set has been proposed as a model for VF [6]. We show that the spiral turbulence associated with VF in this model arises because of spatiotemporal chaos; this is similar to the spatiotemporal chaos in a related model [7] for the catalysis of $\mathrm{CO}$ on $\mathrm{Pt}(110)$ which we have studied elsewhere $[8,9]$. Our defibrillation scheme for the VF model of Ref. [6] relies on the understanding we have developed for spatiotemporally chaotic states in the $\mathrm{CO}$ catalysis model.

Electrical defibrillation entails the application of electrical jolts to the fibrillating heart to make it start beating normally again. Such defibrillation works only about two-thirds of the time and often damages the heart in the process of reviving it [10]. In external defibrillation, electrical shocks $(\simeq 5 \mathrm{kV})$ are applied across the patient's chest; they depolarize all heart cells simultaneously and essentially reset the pacemaking nodes of the heart [11]. Slightly lower voltages $(\simeq 600 \mathrm{~V})$ suffice in open-heart conditions, i.e., when the shock is applied directly on the heart's surface [12]. In our study of the VF model [6] we achieve defibrillation by using very low-amplitude pulses (of order $\mathrm{mV}$ ) applied for a brief duration (of order $100 \mathrm{~ms}$ ) and over a coarse mesh of lines on our model ventricle. Thus, if our defibrillation scheme can be realized in an internal defibrillator, it would constitute a significant advance in these devices.
Before proceeding to our quantitative results it is useful to define the model for ventricular fibrillation [6]. For simplicity we use the model for isotropic cardiac tissue; in this case the equations governing the excitability $e$ and recovery $g$ variables are

$$
\begin{aligned}
& \partial e / \partial t=\nabla^{2} e-f(e)-g, \\
& \partial g / \partial t=\epsilon(e, g)(k e-g) .
\end{aligned}
$$

The function $f(e)$, which specifies fast processes (e.g., the initiation of the action potential) is piecewise linear: $f(e)=C_{1} e$, for $e<e_{1}, f(e)=-C_{2} e+a$, for $e_{1} \leq$ $e \leq e_{2}$, and $f(e)=C_{3}(e-1)$, for $e>e_{2}$. We use the physically appropriate parameter values given in Ref. [6], namely, $e_{1}=0.0026, e_{2}=0.837, C_{1}=20, C_{2}=3$, $C_{3}=15, a=0.06$, and $k=3$. The function $\epsilon(e, g)$, which determines the dynamics of the recovery variable, is $\epsilon(e, g)=\epsilon_{1}$ for $e<e_{2}, \epsilon(e, g)=\epsilon_{2}$ for $e>e_{2}$, and $\epsilon(e, g)=\epsilon_{3}$ for $e<e_{1}$, and $g<g_{1}$ with $g_{1}=1.8$, $\epsilon_{1}=1 / 75, \epsilon_{2}=1.0$, and $\epsilon_{3}=0.3$ (which lies in the range $2>\epsilon_{3}>0.1$ suggested in Ref. [6]). We solve model (1) by using a forward-Euler integration scheme. We discretize our system on a grid of points in space with spacing $\delta x=0.5$ dimensionless units and use the standard five- and seven-point difference stencils [13] for the Laplacians in spatial dimensions $d=2$ and 3, respectively. Our spatial grid consists of a square lattice with $L \times L$ points or cubic lattices with $L \times L \times L_{z}$ points; in our studies we have used values of $L$ ranging from 128 to 512 and $2 \leq L_{z} \leq 16$. Our time step is $\delta t=0.022$ dimensionless units. As in Ref. [6], we define dimensioned time $T$ to be $5 \mathrm{~ms}$ times dimensionless time and 1 spatial unit to be $1 \mathrm{~mm}$, such that the period and wavelength of a spiral wave are approximately $120 \mathrm{~ms}$ and $32.5 \mathrm{~mm}$, respectively. The dimensioned value of the conductivity constant in model (1) is $2 \mathrm{~cm}^{2} / \mathrm{s} \mathrm{[6].} \mathrm{Our}$ initial condition is a broken plane wave which we allow to evolve into a state displaying spiral turbulence (Fig. 1); we control this eventually by our defibrillation scheme. On the edges of our simulation region we use no-flux 

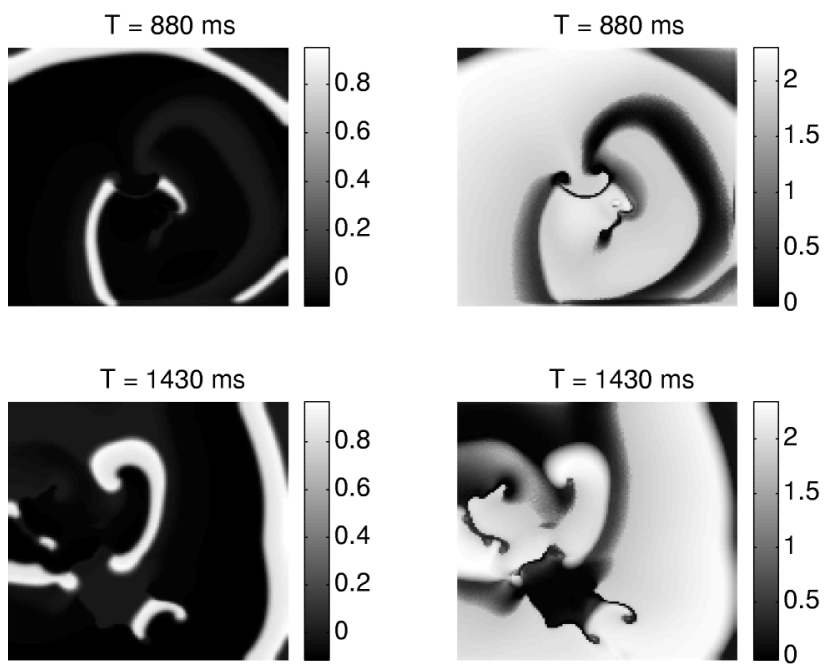

FIG. 1. Pseudo-gray-scale plots showing the evolution of spatiotemporal chaos through spiral breakup in model (1) for physical times $T$ between 880 and $1430 \mathrm{~ms}$. The panels on the left show the excitability $e$ and those on the right the recovery $g$ for all points $(x, y)$ on a two-dimensional $L \times L$ spatial grid with $L=128$. The initial condition used is described in the text.

(Neumann) boundary conditions since the ventricles are electrically insulated from the atria. We impose these boundary conditions numerically by adding an extra layer of grid points on each side of our simulation grid and requiring the values of $e$ to be equal pointwise to their values on the points one layer within the boundary.

Before constructing an efficient defibrillation scheme for VF in model (1) it is important to appreciate the following points: (a) Ventricular fibrillation in this model arises because the system evolves to a state in which large spirals break down $[6,8]$. (b) This state is a long-lived transient whose lifetime $\tau_{L}$ increases rapidly with $L$, the linear size of our system (e.g., for $d=2, \tau_{L} \simeq 850 \mathrm{~ms}$ for $L=100$ whereas $\tau_{L} \simeq 3200 \mathrm{~ms}$ for $L=128$ ). This property of model (1) is in qualitative accord with the experimental finding that the hearts of small mammals are less prone to fibrillation than those of large mammals $[14,15]$. For time $t \gg \tau_{L}$, a quiescent state with $e=g=0$ is obtained. (c) In systems with $L \geqslant 128, \tau_{L}$ is sufficiently long so that a nonequilibrium statistical steady state is established. This state displays spatiotemporal chaos. For example, we find that there are several positive Lyapunov exponents $\lambda_{i}$ (averages for $\lambda_{i}$ are performed for $\tau_{0}<t<\tau_{L}$, where $\tau_{0}$ is the time of decay for initial transients [16]); the number of positive $\lambda_{i}$ increases with $L$ (e.g., the KaplanYorke dimension $D_{\mathrm{KY}}$ [18] increases from 7 to $\gtrsim 35$ as $L$ increases from 128 to 256). (d) Model (1) is akin to a model for the catalysis of $\mathrm{CO}$ on $\mathrm{Pt}(110)$ in so far as both show spiral breakup and spatiotemporal chaos $[6,8]$. Our recent studies of the CO catalysis model [9] have shown that Neumann or no-flux boundary conditions tend to absorb spiral defects and, indeed, the spirals do not last for appreciable periods of time on small systems.
Given that the long transient which leads to VF in model (1) is spatiotemporally chaotic, we might guess that the fields $e$ and $g$ have to be controlled globally to achieve defibrillation. In fact, some earlier studies [19] of spiral breakup in models for ventricular fibrillation have used global control. Here we show that a judicious choice of control points (on a mesh specified below) leads to an efficient defibrillation scheme for model (1). For $d=2$, we divide our simulation domain (of size $L \times L$ ) into $K^{2}$ smaller blocks and choose the mesh size such that it effectively suppresses the formation of spirals. For $d=3$, we use the same control mesh but only on one of the square faces of the $L \times L \times L_{z}$ simulation box. In our defibrillation scheme we apply a pulse to the $e$ field on a mesh composed of lines of width $3 \delta x$. A network of such lines is used to divide the region of simulation into square blocks whose length in each direction is fixed at a constant value $L / K$ for the duration of control. (The blocks adjacent to the boundaries turn out to be rectangular.) The essential point here is that, if a pulse is applied to the $e$ field at all points along the mesh boundaries for a time $\tau_{c}$, then it effectively simulates Neumann boundary conditions (for the block bounded by the mesh) in so far as it absorbs spirals formed inside this block (just as Neumann boundary conditions absorb spiral defects in the $\mathrm{CO}$ catalysis model [9]). Note that $\tau_{c}$ is not large at all since the individual blocks into which the mesh divides our system are of a linear size $L / K$ which is so small that it does not sustain long, spatiotemporally chaotic transients. Nor does $K$, which is related to the mesh density, have to be very large since the transient lifetime, $\tau_{L}$, decreases rapidly with decreasing $L$. We find that, for $d=2, L=128, K=2$, and $\tau_{c}=41.2 \mathrm{~ms}$ is required for defibrillation. In Fig. 2 we illustrate such defibrillation with $\tau_{c}=44 \mathrm{~ms}$. For $d=2$, $L=512, K=8$, and $\tau_{c}=704 \mathrm{~ms}$ suffices. Finally we show that a slight modification of our defibrillation scheme also works for $d=3$ (Fig. 3).

The efficiency of our defibrillation scheme is fairly insensitive to the height of the pulse we apply to the $e$ field along our control mesh so long as this height is above a threshold. To obtain the value of $e$ in $\mathrm{mV}$ units we have scaled the peak amplitude of a spike in the $e$ field (which has an amplitude of 0.9 in dimensionless units) to be equal to $110 \mathrm{mV}$. The latter is a representative average value of the peak voltage of an electrical wave in the heart [14]. With this voltage scaling, dimensioned excitability is computed as $110 / 0.9 \simeq 122.22 \mathrm{mV}$ times dimensionless excitability. We find, e.g., that, for $L=128$, the smallest pulse which yields defibrillation is $57.3 \mathrm{mV} / \mathrm{ms}$ for the parameter values we use; however, we have checked that even stronger pulses (e.g., $278.3 \mathrm{mV} / \mathrm{ms}$ ) also lead to defibrillation. We use a capacitance density of $1 \mu \mathrm{F} / \mathrm{cm}^{2}$ [20], which then yields a current density of $57.3 \mu \mathrm{A} / \mathrm{cm}^{2}$. Note that this threshold is of the order of the smallest potential $\left(\simeq 22.18 \mu \mathrm{A} / \mathrm{cm}^{2}\right)$ required to trigger an actionpotential spike in model (1) with $g=0$; the null clines for 

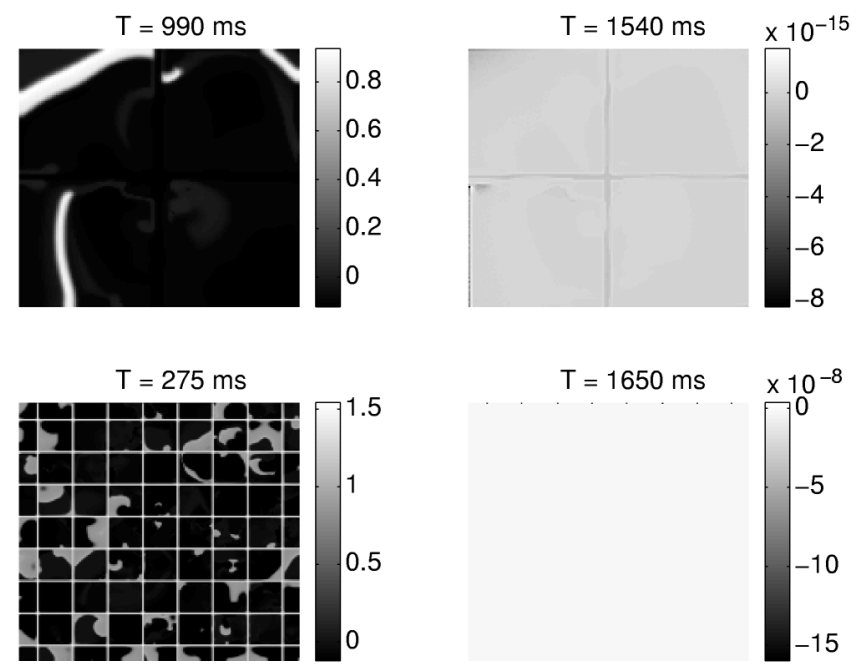

FIG. 2. Pseudo-gray-scale plots of the $e$ field in $d=2$ for $L=128$ (top panels) and $L=512$ (bottom panels) illustrating defibrillation by our control of spiral breakup in model (1). The control mesh divides the domain into four equal squares for $L=128$ and, for $L=512$, into 49 equal squares, 28 equal rectangles along the edges, and 4 small equal squares on the corners. For $L=128$ we apply $57.3 \mu \mathrm{A} / \mathrm{cm}^{2}$ from $T=891 \mathrm{~ms}$ to $T=935 \mathrm{~ms}$ and by $T \gtrsim 1500 \mathrm{~ms}$ spatiotemporal chaos is all but eliminated ( $|e|,|g| \leq 10^{-13}$ at all grid points). For $L=512$, we apply $250 \mu \mathrm{A} / \mathrm{cm}^{2}$ from $T=55 \mathrm{~ms}$ to $T=759 \mathrm{~ms}$ to the $e$ field. By $T=1650 \mathrm{~ms}$, spatiotemporal chaos is all but eliminated ( $|e|,|g| \leq 10^{-7}$ at all grid points) [23].

model (1), in the absence of the Laplacian, are such that this smallest potential increases with increasing $g$. This is physically consistent with the increase in the refractory nature of the heart tissue with increasing $g$.

We have checked that (a) small, local deformations of our control mesh or (b) the angle of the mesh axes with the boundaries do not affect the efficiency of our defibrillation scheme. Furthermore the application of our control pulse on the control mesh does not lead to an instability of the quiescent state; thus it cannot inadvertently promote VF. We have checked specifically that, with $e=0, g=0$ as the initial condition at all spatial points, a wave of activation travels across the system when the control pulse is initiated; this travels quickly ( $\simeq 200 \mathrm{~ms}$ for $L=128$ ) to the boundary where it is absorbed and quiescence is restored. Our defibrillation method also works if $g$ is stimulated instead of $e$. This can be implemented by pharmaceutical means in an actual heart.

Our two-dimensional defibrillation scheme above applies without any change to thin slices of cardiac tissue. However, it is important to investigate whether it can be extended to three dimensions which is clearly required for real ventricles. A naive extension of our mesh into a cubic array of sheets will, of course, succeed in achieving defibrillation. However, such an array of control sheets cannot be easily implanted in a ventricle. We have tried to see, therefore, if we can control the turbulence in a threedimensional version of model (1) on a $L \times L \times L_{z}$ do-
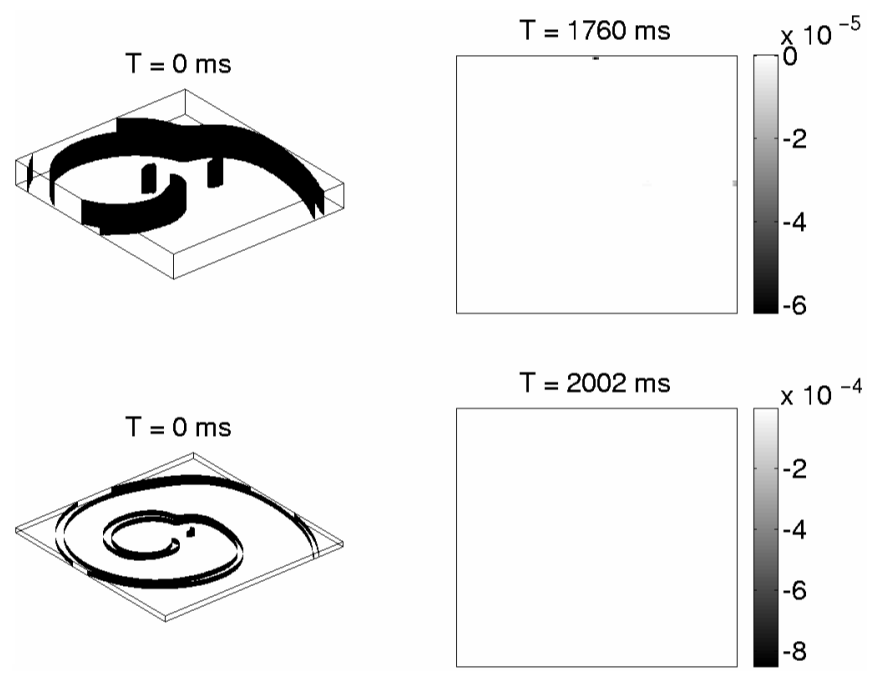

FIG. 3. Isosurface ( $e=0.6)$ plots of initial states (left panels) and pseudo-gray-scale plots of the $e$ field on the top square face (right panels) illustrating defibrillation by the control of spiral breakup in model (1) for $d=3$ : (top panels) $L=128$ and $L_{z}=16$, with the control mesh dividing the top face into 4 equal squares; and (bottom panels) $L=256$ and $L_{z}=8$, with the control mesh dividing the top face into 64 equal squares. In both cases we apply pulses on the control mesh (top face only) of $57.3 \mu \mathrm{A} / \mathrm{cm}^{2}$ with $\tau_{i p}=22 \mathrm{~ms}$ and $\tau_{w}=0.11 \mathrm{~ms}$ (see text). In the former case, with $n=15$, spatiotemporal chaos is all but eliminated by $T=1760 \mathrm{~ms}\left(|e|,|g| \leq 10^{-4}\right.$ at all grid points); in the latter, with $n=30$, it is all but eliminated by $T=2002 \mathrm{~ms}\left(|e|,|g| \leq 10^{-3}\right.$ at all grid points).

main but with the control mesh present only on one $L \times L$ face. For the open faces we use open boundary conditions and for the other faces we use no-flux Neumann boundary conditions. Our defibrillation scheme works if $L_{z} \leq 4$ (we have checked explicitly for $L=220$ ) but not for $L_{z}>4$. A slight modification of this scheme is effective even for $L_{z}>4$ : Instead of applying a pulse for a duration $\tau_{c}$, we apply a sequence of $n$ pulses separated by a time $\tau_{i p}$ and each of duration $\tau_{w}$. We find that, if $L=256$ and $L_{z}=$ 8 , defibrillation occurs in $\simeq 2002 \mathrm{~ms}$ with $\tau_{i p}=22 \mathrm{~ms}$, $\tau_{w}=0.11 \mathrm{~ms}, n=30$, and a control pulse amplitude of $57.3 \mu \mathrm{A} / \mathrm{cm}^{2}$; if $L=128$ and $L_{z}=16$, defibrillation occurs in $\simeq 1760 \mathrm{~ms}$ with $\tau_{i p}=22 \mathrm{~ms}, \tau_{w}=0.11 \mathrm{~ms}$, $n=15$, and a control pulse amplitude of $57.3 \mu \mathrm{A} / \mathrm{cm}^{2}$. We further find that optimal defibrillation is obtained in our model if $\tau_{i p}$ is close to the absolute refractory period for model (1) without the Laplacian term. The efficacy of our control scheme in the three-dimensional case can be understood heuristically as follows: Control via a steady pulse does not work for $L_{z}>4$ since the propagation of this pulse in the $z$ direction (normal to our control mesh) is blocked once the medium in the interior of our simulation domain becomes refractory. However, if we use a sequence of short pulses separated by a time $\tau_{i p}$, then, provided $\tau_{i p}$ is long enough for the medium to recover its excitability, the control-pulse waves can propagate in the $z$ direction and lead to successful defibrillation. 
Typical electrical defibrillation schemes use much higher voltages than in our study. Recent studies [19,21] have explored low-amplitude defibrillation methods in model systems. They constitute an advance over conventional methods, but lack some of the appealing features of our defibrillation scheme. For example, the scheme of Ref. [19] works only when the slow variable (the analog of our $g$ ) is controlled; though this can be done, in principle, by pharmaceutical means, it is clearly less direct than control via electrical means. Reference [21] uses electrical defibrillation, but has been demonstrated to prevent only one spiral from breaking up, as opposed to the suppression of a spatiotemporally chaotic state with broken spirals by our defibrillation scheme. We have checked explicitly that, for the spatiotemporally chaotic state of model (1), a straightforward implementation of the defibrillation scheme of Ref. [21] is ineffective. \{This scheme applies pulses to the fast variable [ $e$ in model (1)] on a two-dimensional, discrete lattice of points.\} The control current density in Ref. [21] is comparable to our $\simeq 57.3 \mu \mathrm{A} / \mathrm{cm}^{2}$, which is much lower than $139 \mu \mathrm{A} / \mathrm{cm}^{2}$, the maximum value of the ionic current during depolarization in the Beeler-Reuter model.

We have checked that our defibrillation scheme is not sensitively model dependent. For example, we have used the same scheme to eliminate spatiotemporal chaos associated with spiral breakup in the model for the catalysis of $\mathrm{CO}$ on $\operatorname{Pt}(110)$ mentioned above $[7,8]$. We have found recently that our defibrillation scheme also works for the biologically realistic Beeler-Reuter model $[20,22]$ for VF.

In conclusion, then, we have developed an efficient method for defibrillation by the elimination of spiral turbulence in model (1). Our method has the attractive feature that it uses very low-amplitude pulses, applied only for a short duration on a coarse control mesh of lines. And, to the best of our knowledge, our study is the only one which shows how to attain defibrillation by the control of spatiotemporal chaos and spiral turbulence in a model for VF in both two and three dimensions. We hope our work will stimulate experimental tests of the efficacy of our defibrillation method.

We thank CSIR (India) for support, SERC (IISc, Bangalore) for computational facilities, and A. Pumir and N. I. Subramanya for discussions.

[1] A. T. Winfree, Chaos 8, 1 (1998). Focus issue on fibrillation in normal ventricular myocardium [Chaos 8 (1998)].
[2] American Heart Disease Statistics on the World Wide Web (URL: http://sln.fi.edu/biosci/healthy/ stats.html).

[3] Computational Biology of the Heart, edited by A. V. Panfilov and A. V. Holden (Wiley, Chichester, 1997).

[4] J. Jalife, R. A. Gray, G. E. Morley, and J. M. Davidenko, Chaos 8, 79 (1998).

[5] R. A. Gray, A. M. Pertsov, and J. Jalife, Nature (London) 392, 75 (1998); F. X. Witkowski, L. J. Leon, P. A. Penkoske, W. R. Giles, M. L. Spano, W. L. Ditto, and A. T. Winfree, Nature (London) 392, 78 (1998).

[6] A. V. Panfilov and P. Hogeweg, Phys. Lett. A 176, 295 (1993); A. V. Panfilov, Chaos 8, 57 (1998).

[7] M. Hildebrand, M. Bär, and M. Eiswirth, Phys. Rev. Lett. 75, 1503 (1995).

[8] A. Pande, S. Sinha, and R. Pandit, J. Indian Inst. Sci. 79, 31 (1999).

[9] A. Pande and R. Pandit, Phys. Rev. E 61, 6448 (2000).

[10] R. Pool, Science 247, 1294 (1990).

[11] M.S. Eisenberg, L. Bergner, A.P. Hallstrom, and R. O. Cummins, Sci. Am. 254, No. 5, 25 (1986).

[12] L. W. Piller, Electronic Instrumentation Theory of Cardiac Technology (Staples Press, London, 1970).

[13] W. H. Press, S. A. Teukolsky, W. T. Vetterling, and B.P. Flannery, Numerical Recipes in C (Cambridge University Press, London, 1995).

[14] A. T. Winfree, When Time Breaks Down (Princeton University Press, Princeton, 1987).

[15] Y.-H. Kim, A. Garfinkel, T. Ikeda, T.-J. Wu, C. A. Athill, J. N. Weiss, H. S. Karagueuzian, and P.-S. Chen, J. Clin. Invest. 100, 2486 (1997).

[16] We use standard algorithms [8,17] for calculating the first $N$ Lyapunov exponents with $N=35$ in most of our studies.

[17] T. Parker and L. O. Chua, Practical Numerical Algorithms for Chaotic Systems (Springer, Berlin, 1989).

[18] We calculate the Kaplan-Yorke dimension [17] as follows: Label the calculated Lyapunov exponents $\lambda_{i}$ in decreasing order such that $\lambda_{1} \geq \lambda_{2} \geq \lambda_{3} \cdots \geq \lambda_{N}$. Now define $D$ as that index at which $\sum_{i=1}^{D} \lambda_{i} \geq 0$ and $\sum_{i=1}^{D+1} \lambda_{i}<0$. Then the Kaplan-Yorke dimension $D_{\mathrm{KY}}$ is $D_{\mathrm{KY}}=D+$ $\left(\sum_{i=1}^{D} \lambda_{i}\right) /\left|\lambda_{D+1}\right|$.

[19] G. V. Osipov and J. J. Collins, Phys. Rev. E 60, 54 (1999).

[20] G. W. Beeler and H. Reuter, J. Physiol. 268, 177 (1977).

[21] W.-J. Rappel, F. Fenton, and A. Karma, Phys. Rev. Lett. 83, 456 (1999).

[22] S. Sinha, A. Sen, A. Pande, and R. Pandit (to be published).

[23] See http://theory1.physics.iisc.ernet.in/rahul/images/ heart.mpg for an animated figure. 